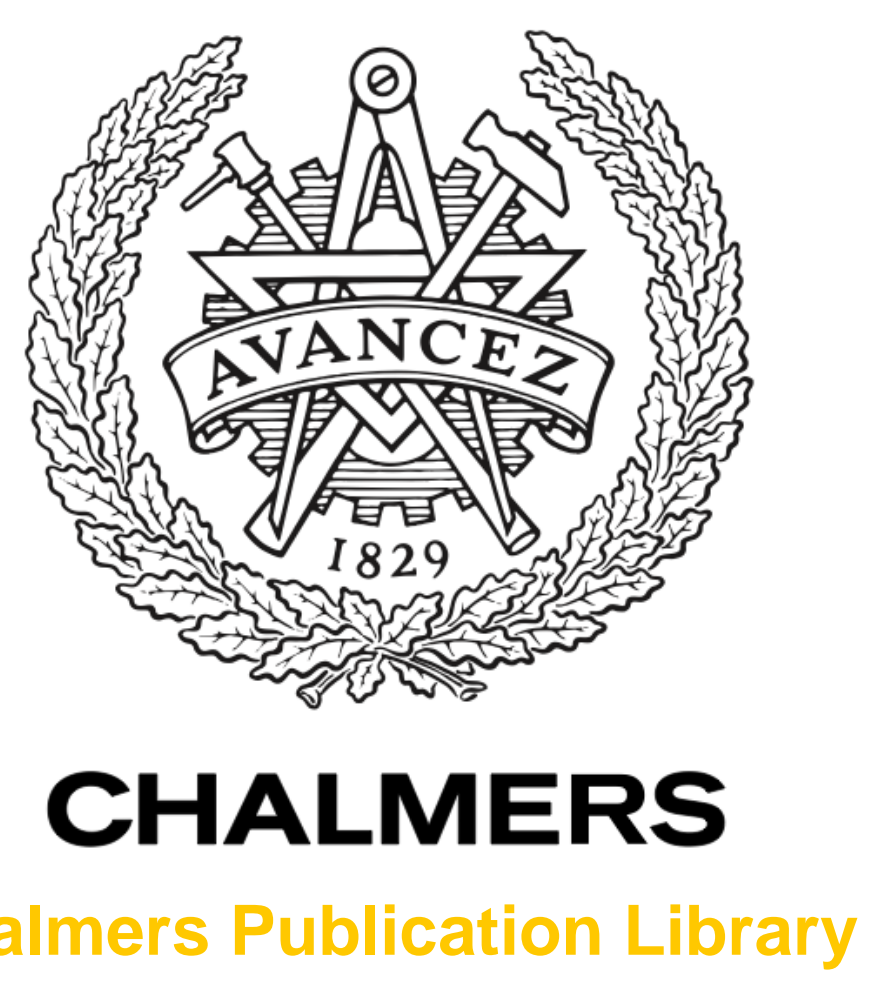

Chalmers Publication Library

A model-based analysis of phase jitter in RF oscillators

This document has been downloaded from Chalmers Publication Library (CPL). It is the author's version of a work that was accepted for publication in:

Proceedings - 66th IEEE International Frequency Control Symposium, IFCS 2012, Baltimore, 21-24 May 2012 (ISSN: 1075-6787)

Citation for the published paper:

Khanzadi, M. ; Panahi, A. ; Kuylenstierna, D. (2012) "A model-based analysis of phase jitter in RF oscillators". Proceedings - 66th IEEE International Frequency Control Symposium, IFCS 2012, Baltimore, 21-24 May 2012 pp. 508-511.

http://dx.doi.org/10.1109/FCS.2012.6243677

Downloaded from: http://publications.lib.chalmers.se/publication/157578

Notice: Changes introduced as a result of publishing processes such as copy-editing and formatting may not be reflected in this document. For a definitive version of this work, please refer to the published source. Please note that access to the published version might require a subscription.

Chalmers Publication Library (CPL) offers the possibility of retrieving research publications produced at Chalmers University of Technology. It covers all types of publications: articles, dissertations, licentiate theses, masters theses, conference papers, reports etc. Since 2006 it is the official tool for Chalmers official publication statistics. To ensure that Chalmers research results are disseminated as widely as possible, an Open Access Policy has been adopted.

The CPL service is administrated and maintained by Chalmers Library. 


\title{
A model-based analysis of phase jitter in RF oscillators
}

\author{
M. Reza Khanzadi ${ }^{\dagger}, *$, Ashkan Panahi*, Dan Kuylenstierna ${ }^{\dagger}$, Thomas Eriksson ${ }^{*}$ \\ $\dagger$ Department of Microtechnology and Nanoscience, *Department of Signals and Systems \\ Chalmers University of Technology, Gothenburg, Sweden \\ khanzadi@chalmers.se
}

\begin{abstract}
The closed-form autocorrelation function of the phase jitter accumulation process in presence of $1 / f^{3}$ and $1 / f^{2}$ shape noises is derived from the single-sideband (SSB) phase noise $(P N)$ measurements. Exploiting the calculated autocorrelation function, a lower bound for the minimum achievable mean square error $(M S E)$ of the $\mathrm{PN}$ prediction in a typical single-input singleoutput communication system is computed. This bound links the performance of a communication system suffering from the PN directly to the SSB PN measurements.
\end{abstract}

Index Terms-Phase Noise, Phase Jitter, Phase Estimation, RF Oscillator, Flicker Noise, Wiener Phase Noise, SSB, CRLB.

\section{INTRODUCTION}

Phase noise $(P N)$ in the output of the oscillators deteriorates the performance of the communication systems. In order to effectively estimate and compensate the PN, models that accurately capture the characteristics of non-ideal oscillators are required. PN modeling has been investigated extensively in the circuits and systems, and microwave community over the past decades [1]-[7]. However, despite this progress in proposing more realistic models for the random $\mathrm{PN}$, incomprehensive models are exploited in the communication society. As a result, the connection between the true PN models and the performance of the systems employing the noisy oscillators is still lacking.

One example of such models is the Wiener PN that in general cannot fully resemble the properties of the time varying PN (e.g., [8], [9]). The Wiener model is developed according to the Lorentzian portion of the single-sideband (SSB) PN spectrum, which has a $1 / f^{2}$ shape. The corner frequency between $1 / f^{3}$ and $1 / f^{2}$ portions of the SSB, $f_{c}$, for low speed (low frequency) oscillators is usually quiet low. Therefore, the effect of $1 / f^{3}$ is almost negligible even after using narrow bandwidth phase locked loops (PLLs) [6]. High speed oscillators normally have $f_{c}$ located in a higher frequency, which makes it impossible to eliminate the effect of $1 / f^{3}$ noise [6], [10].

PN with $1 / f^{3}$ and $1 / f^{2}$-shaped SSB is resulted from the accumulation of random noise over the time and hence it has a cumulative nature. In this paper, a closed-form autocorrelation function $(A C F)$ for the $\mathrm{PN}$ accumulation process due to $1 / f^{3}$ and $1 / f^{2}$ noises is derived. Then, a lower bound for the mean square error (MSE) of the PN prediction in the receiver side of a communication system affected by the superposition of $1 / f^{3}$ and $1 / f^{2}$ phase noises is computed. This bound is a function of the SSB PN measurements and can be used to evaluate the effect of using oscillators with different PN characteristics on the performance of the communication systems.

In the next section, a system model of the considered communication system is introduced. In Sec. III, the source of $\mathrm{PN}$ in the oscillator is studied and a closed form ACF for the PN accumulation process is derived. In Sec. IV, a lower bound on estimation (prediction) of the PN in a typical communication system is derived. This bound is computed for two different measurements in the Sec. V and finally the paper is concluded in Sec. VI.

Notations: Italic letters $(x)$ are scalar variables, bold letters $(\mathbf{x})$ are vectors, bold upper case letters $(\mathbf{X})$ are matrices, $\left([\mathbf{X}]_{a, b}\right)$ is the $(a, b)^{t h}$ entry of matrix $\mathbf{X}, \mathbb{E}[\cdot]$ denotes the statistical expectation, $\Im(\cdot)$ and $\arg (\cdot)$ represent the image and angle of complex values, respectively, and $(\cdot)^{T}$ denotes the matrix or vector transpose.

\section{SySTEM MODEL}

The complex baseband received signal of a communication system at time instant $n$ after removing the data dependency can be written as

$$
r(n)=e^{j \phi(n)}+w(n)
$$

where $e^{j \phi(n)}$ represents the phasor of $\phi(n)$, which is the unwanted phase fluctuation of the $n$th received symbol, and $w(n)$ is the zero-mean complex additive white Gaussian noise $(A W G N)$ with variance $\sigma_{w}^{2} . \phi(n)$ represents the superposition of the PN from the transmitter and receiver oscillators in the discrete-time domain.

Assuming a high signal to noise ratio (SNR) scenario and taking the same steps as [11], the argument of received signal can be determined as

$$
\psi(n) \triangleq \arg (r(n)) \approx \phi(n)+\dot{w}(n)
$$

where $\dot{w}(n) \triangleq \Im(w(n))$ is a real zero-mean Gaussian distributed noise with variance $\sigma_{w}^{2}=\frac{\sigma_{w}^{2}}{2}$.

\section{Phase NOISE}

In order to understand the effect of $\mathrm{PN}$ on the system performance, it is necessary to study the source of this phenomenon. The output of real sinusoidal oscillators in the continuous-time domain can be written as

$$
V(t)=A(t) \cos \left(2 \pi f_{0} t+\phi(t)\right),
$$

where $f_{0}$ is the oscillator's central frequency. $A(t)$ and $\phi(t)$ are amplitude noise and phase noise, respectively and are modeled 


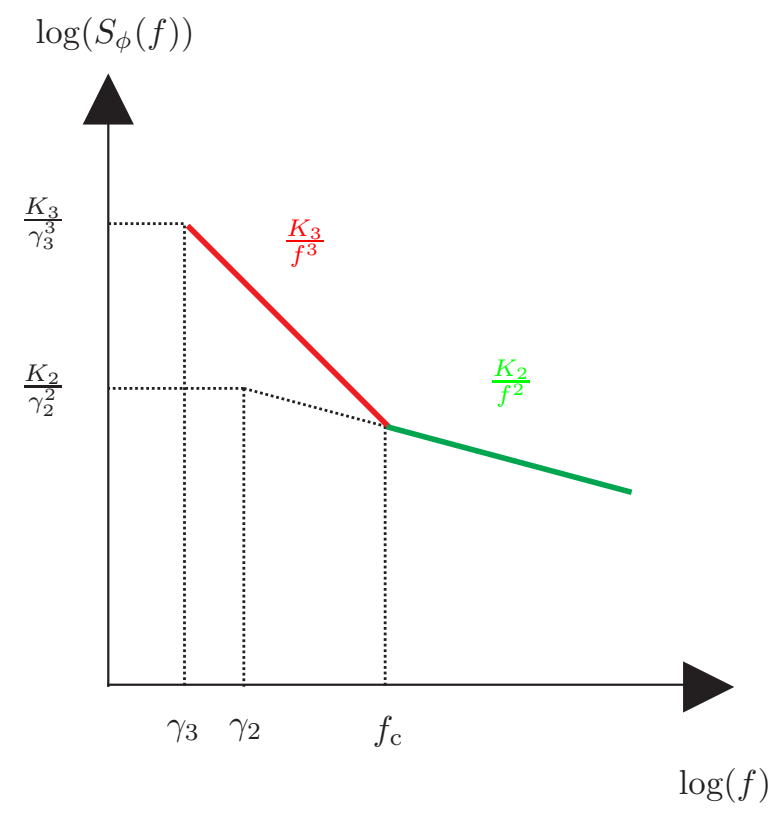

Fig. 1. Phase Noise PSD with introduced cut-off frequencies. $S_{\phi}(f) \approx$ $\mathcal{L}_{\phi}(f)$, where $\mathcal{L}_{\phi}(f)$ is the phase noise SSB. $K_{3}$ and $K_{2}$ are frequency figures of merit. $\gamma_{3}$ and $\gamma_{2}$ denote the low cut-off frequencies.

as two independent random processes (RPs). It is shown in [4] that for a frequency range close to the central frequency of the oscillator, compared to the phase noise, the amplitude noise has an insignificant effect on the output signal of the oscillator.

Fig. 1 shows the power spectral density (PSD) of the PN. As discussed in [6], two low cut-off frequencies are introduced for $1 / f^{3}$ and $1 / f^{2}$ noises to support the wide sense stationary (WSS) characteristic of the PN. In practice, when a limitedtime record of the PN process is available, the statistical properties of the PN process, such as mean and variance cannot change dramatically over this short record. For example, the recording length limitation of the measurement instrument is discussed as a support for this assumption in [6], [12]. The packet-based communication is another practical example for which this assumption is valid. In this case, information is transmitted over the limited length packets that are processed independently in the receiver. Thus, the PN process that affects the transmitted packets can be assumed WSS over each block due to the limited transmission time.

Accumulation of the PN over the time can be modeled as a $\mathrm{RP}$ which is called jitter accumulation process $(J A P)$ here. The continuous-time JAP, $\zeta(t)$, is defined as accumulation process of the PN over the time delay $\Delta T$.

$$
\zeta(t, \Delta T)=\phi(t)-\phi(t-\Delta T) .
$$

JAP is a stationary RP with zero-mean Gaussian distribution and as discussed in [6], the procedure of finding the samples of this process can be expressed by a linear time invariant (LTI) sampling system with impulse response of

$$
h(t)=\delta(t)-\delta(t-\Delta T) .
$$

The ACF of the JAP, which is the inverse Fourier transform of the JAP's PSD can be determined as

$$
R_{\zeta}(\tau)=\int_{-\infty}^{+\infty} S_{\phi}(f)|H(j 2 \pi f)|^{2} e^{2 \pi f \tau} \mathrm{d} f,
$$

where $H(j 2 \pi f)=1-e^{-j 2 \pi f \Delta T}$ is the frequency response of (5) and $\tau$ is the time lag parameter. (6) can be simplified further to

$$
R_{\zeta}(\tau)=8 \int_{0}^{+\infty} S_{\phi}(f) \sin (\pi f \Delta T)^{2} \cos (2 \pi f \tau) \mathrm{d} f,
$$

that gives the continuous-time ACF of the JAP for a given PN process PSD, $S_{\phi}(f)$.

Considering the cut-off frequency $\gamma_{2}$, PSD of $\phi_{2}(t)$ resembling $1 / f^{2}$ noise is written as

$$
S_{\phi_{2}}(f)=\frac{K_{2}}{f^{2}+\gamma_{2}^{2}}
$$

where $K_{2}$ is called $1 / f^{2}$ frequency figure of merit [6]. The ACF of JAP can be calculated from (7) and (8) as

$$
R_{\zeta_{2}}(\tau) \approx \begin{cases}4 K_{2} \pi^{2} \Delta T & \text { if } \tau=0 \\ 0 & \text { otherwise }\end{cases}
$$

Here, the ACF is not a function of cut-off frequency, $\gamma_{2}$. This result also shows the JAP samples are uncorrelated with each other. Thus, in presence of only $\phi_{2}(t)$, variance of JAP is a sufficient statistic to describe this zero-mean process. $R_{\zeta_{2}}(\tau=$ $0)$, variance of the JAP, is proportional to the time delay $\Delta T$ which has been discussed in the literature (e.g., [2], [6], [13]).

$\phi_{3}(t)$ is defined as a $\mathrm{RP}$ resembling $1 / f^{3}$ noise which is WSS over a finite time interval. A low cut-off frequency is defined for $1 / f^{3}$ noise PSD which is assumed to be flat below this cut-off frequency. Hence, the PN PSD is determined as

$$
S_{\phi_{3}}(f)=\frac{K_{3}}{|f|^{3}+\gamma_{3}^{3}},
$$

where $K_{3}$ and $\gamma_{3}$ are the $1 / f^{3}$ frequency figure of merit and introduced cut-off frequency, respectively. Using (7) and (10), the ACF of JAP can be computed by solving the following integral:

$$
R_{\zeta_{3}}(\tau)=8 \int_{0}^{+\infty} \frac{K_{3}}{f^{3}+\gamma_{3}^{3}} \sin (\pi f \Delta T)^{2} \cos (2 \pi f \tau) \mathrm{d} f .
$$

Following a set of algebraic manipulations, the ACF is approximated as

$$
\begin{aligned}
& R_{\zeta_{3}}(\tau=0)=-8 K_{3} \pi^{2} \Delta T^{2}\left(\Lambda+\log \left(2 \pi \gamma_{3} \Delta T\right)\right) \\
& R_{\zeta_{3}}(\tau=|\Delta T|)=-8 K_{3} \pi^{2} \Delta T^{2}\left(\Lambda+\log \left(8 \pi \gamma_{3} \Delta T\right)\right)
\end{aligned}
$$

otherwise

$$
\begin{aligned}
R_{\zeta_{3}}(\tau)= & -8 K_{3} \pi^{2}\left[-\tau^{2}\left(\Lambda+\log \left(2 \pi \gamma_{3}|\tau|\right)\right)\right. \\
& +\frac{(\tau+\Delta T)^{2}}{2}\left(\Lambda+\log \left(2 \pi \gamma_{3}|\tau+\Delta T|\right)\right) \\
& \left.+\frac{(\tau-\Delta T)^{2}}{2}\left(\Lambda+\log \left(2 \pi \gamma_{3}|\tau-\Delta T|\right)\right)\right]
\end{aligned}
$$


where $\Lambda \triangleq \Gamma-\frac{3}{2}$, and $\Gamma \approx 0.5772$ is the Euler-Mascheroni constant. The calculated variance of JAP, $R_{\zeta_{2}}(\tau=0)$, is almost proportional to $\Delta T^{2}$ which is similar to the results of [6], [13]. It is clear that in contrast to $\phi_{2}(t)$, the JAP samples are correlated in this case. It means, looking at the variance of JAP alone is not adequate to judge the behavior of the oscillator in a system; in presence of $1 / f^{3}$ noise, it is necessary to incorporate the correlation properties of the JAP samples.

Finally, according to the mutually independence assumption of $\phi_{2}(t)$ and $\phi_{3}(t)$, the total ACF of JAP is computed as

$$
R_{\zeta}(\tau)=R_{\zeta_{2}}(\tau)+R_{\zeta_{3}}(\tau) .
$$

$R_{\zeta}(\tau)$ is employed in the next section to compute a performance measure for the PN affected communication systems.

\section{Phase Noise Estimation Performance}

In this section, a lower bound for the MSE of the phase estimation is computed. It is assumed that the carrier phase recovery is performed in the digital domain. Assume $\Delta T$, introduced in Sec. III, is the time duration of each transmitted symbol. As a result, sampling (4) gives

$$
\zeta(n)=\phi(n+1)-\phi(n)
$$

where $\zeta(n)$ is the discrete time JAP that is the phase accumulation process over each transmitted symbol. The ACF of discrete-time JAP can be computed by sampling (13). To do so, the time difference $\tau$ is replaced with $l \Delta T$, where $l$ represents the discrete time lag or distance index between the symbols.

Here, a scenario for estimation (prediction) of $n^{\text {th }}$ received symbol's PN, from the argument vector of $N$ previous observation symbols, $\psi(n) \triangleq[\psi(n-1), \ldots, \psi(n-N)]^{T}$, is studied. Employing (2) and the recursive model in (14), phase of any observation symbol at time $(n-x)$ prior to time $n$ can be written as

$$
\psi(n-x)=\phi(n)-\sum_{j=0}^{x-1} \zeta(n-j)+\dot{w}(n-x) .
$$

$\zeta(n)$ and $\dot{w}(n)$ have real zero-mean Gaussian distribution. Consequently, $\boldsymbol{\psi}(n)$ has an $N$-variate Gaussian distribution as follows:

$$
f(\boldsymbol{\psi}(n) \mid \phi(n))=\frac{e^{\left[-\frac{1}{2}(\boldsymbol{\psi}(n)-\mathbf{m})^{T} \mathbf{C}^{-1}(\boldsymbol{\psi}(n)-\mathbf{m})\right]}}{\sqrt{\left((2 \pi)^{N} \operatorname{det}(\mathbf{C})\right)}},
$$

where $\mathbf{m} \triangleq \phi(n) \mathbf{1}^{N \times 1}$ denotes the mean vector and $\mathbf{C}$ is the covariance matrix with elements

$$
[\mathbf{C}]_{x, y}=\sum_{j=0}^{x-1} \sum_{k=0}^{y-1} R_{\zeta}(j-k)+\delta(x-y) \frac{\sigma_{w}^{2}}{2}, \quad x, y=1 \ldots N .
$$

Assume the best unbiased estimator is used in the receiver. The best estimator, here refers to the one that has the lowest MSE of the estimation. The minimum MSE among unbiased estimators can be bounded using the Cramér-Rao lower bound
$(C R L B)$ [14]. Defining $\ell(\phi(n)) \triangleq \log (f(\psi(n) \mid \phi(n)))$ and denoting the estimated PN as $\hat{\phi}(n)$, the CRLB can be computed as

$$
\mathbb{E}\left[(\phi(n)-\hat{\phi}(n))^{2}\right] \geq\left(\mathbb{E}\left[-\frac{\partial^{2} \ell(\phi(n))}{\partial \phi(n)^{2}}\right]\right)^{-1},
$$

where in the current case the minimum variance of error becomes:

$$
\mathbb{E}\left[(\phi(n)-\hat{\phi}(n))^{2}\right] \geq \frac{1}{\mathbf{1}^{T} \mathbf{C}^{-1} \mathbf{1}} .
$$

\section{EXPERIMENTAL RESULTS}

The proposed MSE bound can be used in order to predict the system performance for any given PN measurements. The required parameters are extracted from the real measurements of the oscillators to compute the MSE bound and evaluate which oscillator can perform better in a communication system.

For experimental demonstration of the theory presented in Sec. IV, a $9.9 \mathrm{GHz}$ balanced Colpitts oscillator implemented in GaN HEMT MMIC technology is used [15]. This oscillator has a SSB PN spectra that changes considerably with bias condition. Fig. 2 and Fig. 3 show the measured SSB PN at two different bias points. The $1 / f^{3}$ noise level in the measurement of Fig. 3 is almost 10 times higher than that of Fig. 2 and $1 / f^{2}$ noise is almost negligible in the Fig. 3 measurement. Fig. 4 compares the calculated MSE bounds for the given measurements. As seen in this figure, a lower PN estimation MSE can be achieved by using the first oscillator due to the lower level of $1 / f^{3}$ noise.

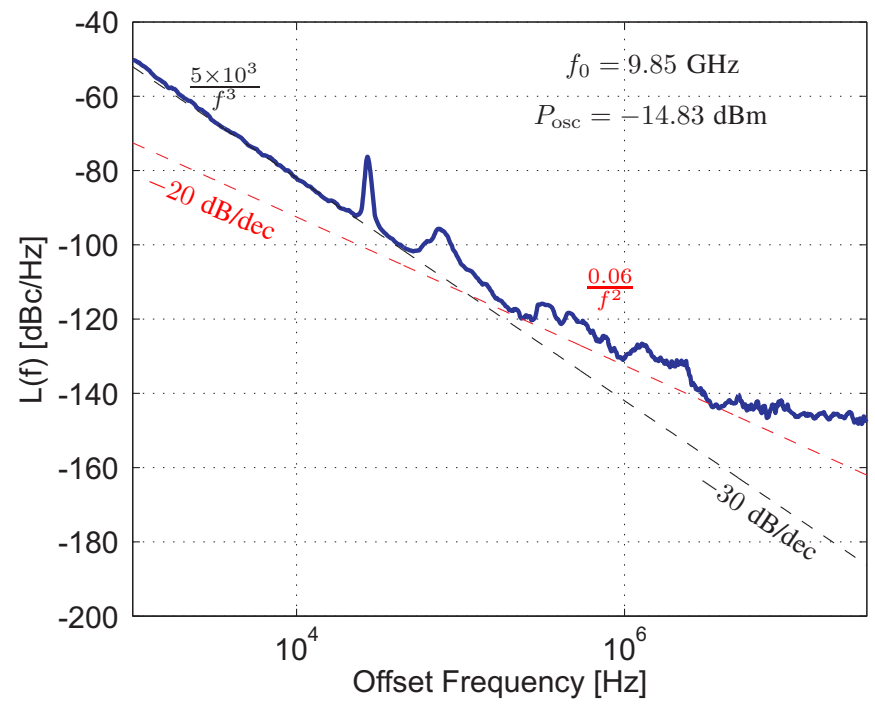

Fig. 2. First oscillator: $K_{3}=5 \times 10^{3}, K_{2}=0.06, f_{c}=83.3 \mathrm{kHz}$.

\section{CONCLUSION}

A closed form ACF for the accumulated jitter due to $1 / f^{3}$ and $1 / f^{2}$ noises is computed. Employing the calculated ACF, a lower bound for estimation of the PN has been derived. The proposed bound is a function of SSB PN measurements and 


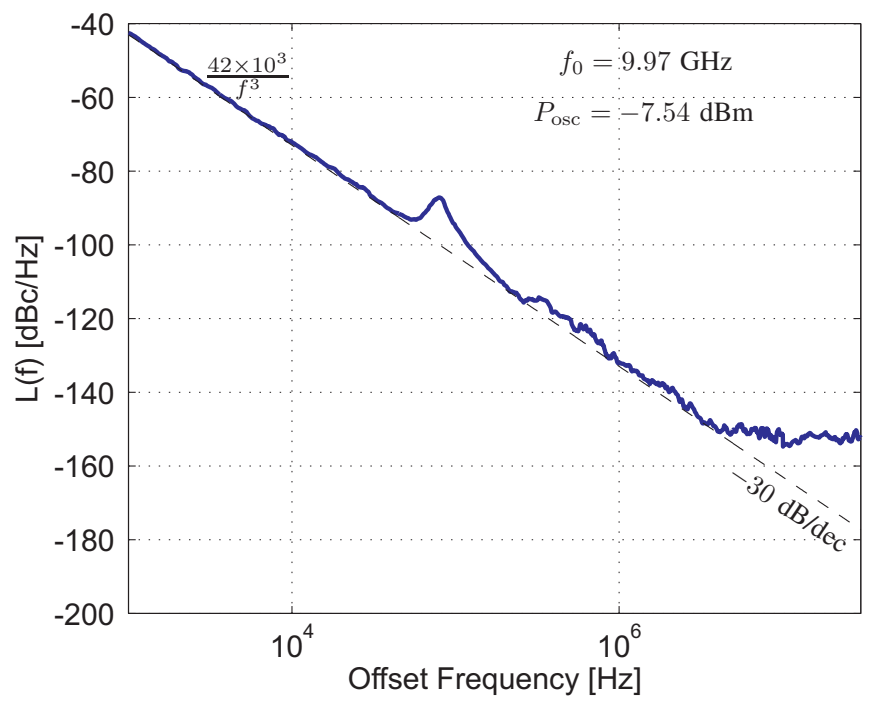

Fig. 3. Second oscillator: $K_{3}=42 \times 10^{3}, 1 / f^{2}$ is almost negligible.

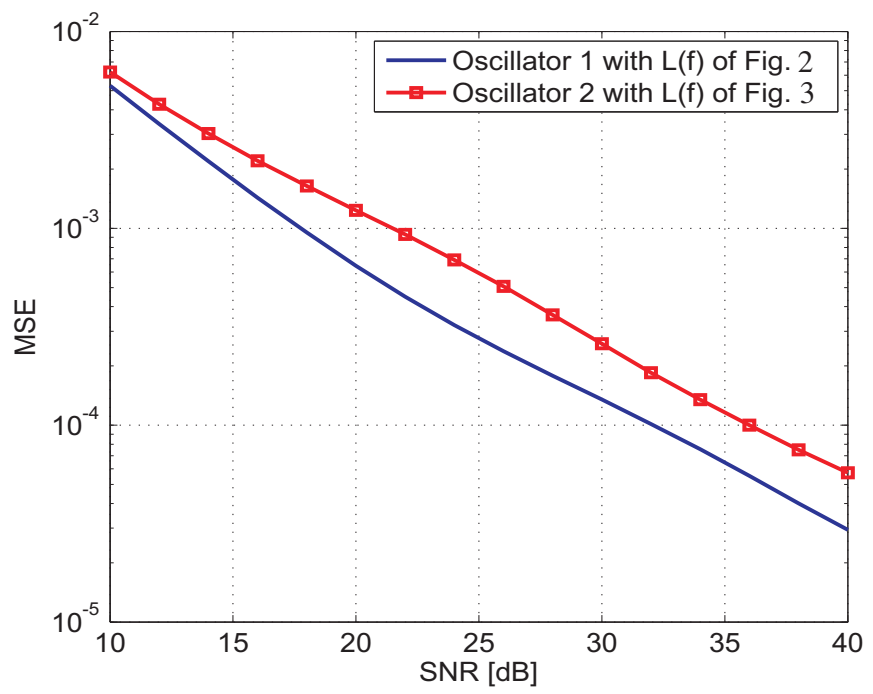

Fig. 4. MSE bound comparison of given measurements in Fig. 2 and 3. The low cut-off frequency, $\gamma_{3}$, is considered to be $1 \mathrm{~Hz}$, symbol rate is $10^{6}[\mathrm{Symbol} / \mathrm{s}]$, and block-length, $N$, is set to 10 .

can be used to compare the effect of using different oscillators on the performance of the communication systems.

\section{REFERENCES}

[1] D. Leeson, "A simple model of feedback oscillator noise spectrum," Proc. IEEE, vol. 54, no. 2, pp. 329 - 330, Feb. 1966.

[2] J. McNeill, "Jitter in ring oscillators," IEEE J. Solid-State Circuits, vol. 32, no. 6, pp. $870-879$, jun 1997.

[3] A. Hajimiri and T. Lee, "A general theory of phase noise in electrical oscillators," IEEE J. Solid-State Circuits, vol. 33, no. 2, pp. 179 -194, Feb. 1998.

[4] G. Klimovitch, "A nonlinear theory of near-carrier phase noise in freerunning oscillators," in Proc. IEEE Intl. Conf. on Circuits Systs., Mar. 2000, pp. $1-6$.

[5] A. Demir, A. Mehrotra, and J. Roychowdhury, "Phase noise in oscillators: a unifying theory and numerical methods for characterization," IEEE Trans. Circuits Syst. I, Fundam. Theory Appl., vol. 47, no. 5, pp. $655-674$, May 2000.
[6] C. Liu and J. McNeill, "Jitter in oscillators with $1 / f$ noise sources," in Proc. IEEE 2004 Intl. Symp. on Circuits Systs., vol. 1, Vancouver, BC, Canada, may 2004, pp. 773-776.

[7] A. Chorti and M. Brookes, "A spectral model for RF oscillators with power-law phase noise," IEEE Trans. Circuits Syst. I, Reg. Papers, vol. 53, no. 9, pp. $1989-1999$, Sep. 2006.

[8] L. Tomba, "On the effect of wiener phase noise in OFDM systems," IEEE Trans. Commun., vol. 46, no. 5, pp. 580 -583, May 1998.

[9] R. Krishnan, M.R. Khanzadi, L. Svensson, T. Eriksson, and T. Svensson, "Variational bayesian framework for receiver design in the presence of phase noise in MIMO systems," in 2012 IEEE Wireless Communications and Networking Conference, Apr. 2012, pp. 1 -6.

[10] C. Liu and J. McNeill, "Jitter in deep submicron CMOS single-ended ring oscillators," in ASIC, 2003. Proc. 5th Intl. Conf. on, vol. 2, oct. 2003, pp. $715-718$ Vol.2.

[11] M.R. Khanzadi, H. Mehrpouyan, E. Alpman, T. Svensson, D. Kuylenstierna, and T. Eriksson, "On models, bounds, and estimation algorithms for time-varying phase noise," in Signal Processing and Communication Systems (ICSPCS), 2011 5th International Conference on, dec. 2011, pp. $1-8$.

[12] J. McNeill, "Jitter in ring oscillators," Ph.D. dissertation, Boston University, 1994.

[13] A. Demir, "Computing timing jitter from phase noise spectra for oscillators and phase-locked loops with white and 1/f noise," IEEE Trans. Circuits Syst. I, Reg. Papers, vol. 53, no. 9, pp. $1869-1884$ sept. 2006.

[14] S. M. Kay, Fundamentals of Statistical Signal Processing, Estimation Theory. Prentice Hall, Signal Processing Series, 1993.

[15] D. Kuylenstierna, S. Lai, H. Zirath, I. Angelov, and R. Rorsman, "A low phase-noise GaN HEMT MMIC oscillator," Submitted to IEEE Microw. Wireless Compon. Lett., 2012. 